\title{
Man Portable Acoustic Navigation Buoys
}

\author{
Rui Almeida \\ INESC TEC \\ and FEUP DEEC \\ rui.almeida@fe.up.pt
}

\author{
Nuno Cruz \\ INESC TEC \\ and FEUP DEEC \\ nacruz@fe.up.pt
}

\author{
Aníbal Matos \\ INESC TEC \\ and FEUP DEEC \\ anibal@fe.up.pt
}

\begin{abstract}
This paper presents a new generation of man portable acoustic navigation buoys. The aim of these buoys is to facilitate the deployment of an underwater acoustic positioning system for the operation of Autonomous Underwater Vehicles. Each buoy includes only the vital modules required for the most typical schemes of underwater acoustic navigation, packed in a small but dynamically stable platform for one day long operations in coastal waters. We will present an overview of the systems hardware and electronics, and also the key features of the deployment and operation of the beacons.
\end{abstract}

\section{INTRODUCTION}

One of the key challenges in the operation of autonomous vehicles is the computation, in real time, of an accurate estimate of the vehicle's position. Such an estimate is fundamental not only to feed the navigation and control systems, but also to provide a geo-reference to all data collected during a mission. In the case of underwater systems, this challenge is particularly demanding due to the strong attenuation of electromagnetic signals, which inhibit the use of ubiquitous solutions based on GNSS or other radio signals. Moreover, these difficulties naturally extend to the exchange of information between the autonomous vehicles and any supervisor, which means that tracking these vehicles during a mission can be another, maybe independent, challenge. Luckily, the attenuation of acoustic waves is much lesser underwater than over the air. Therefore, many localization and tracking methods have been proposed, based on the exchange of simple acoustic signals between the vehicles and a set of acoustic beacons deployed in the operation area.

The development of these new buoys at INESC TEC follows a long term line of research and development in underwater acoustic positioning based in modular systems for transmitting and detecting underwater signals. In [1], the first beacons were built with the ability to work as transponders and also to passively track AUVs, and they were deployed from large moored buoys. Later, there was a redesign to incorporate time synchronization based on GPS signals and NTP, and also to integrate the acoustic beacons in smaller buoys to act as portable coastal observatories for short term deployments [2]. These developments have successfully supported multiple operations of AUVs for the last 15 years. Around the world, similar efforts have been made to provide absolute position to AUVs while they navigate underwater, in mechanisms sometimes referred to as underwater GPS [3]. However, as the size of AUVs gets smaller and they require less logistics

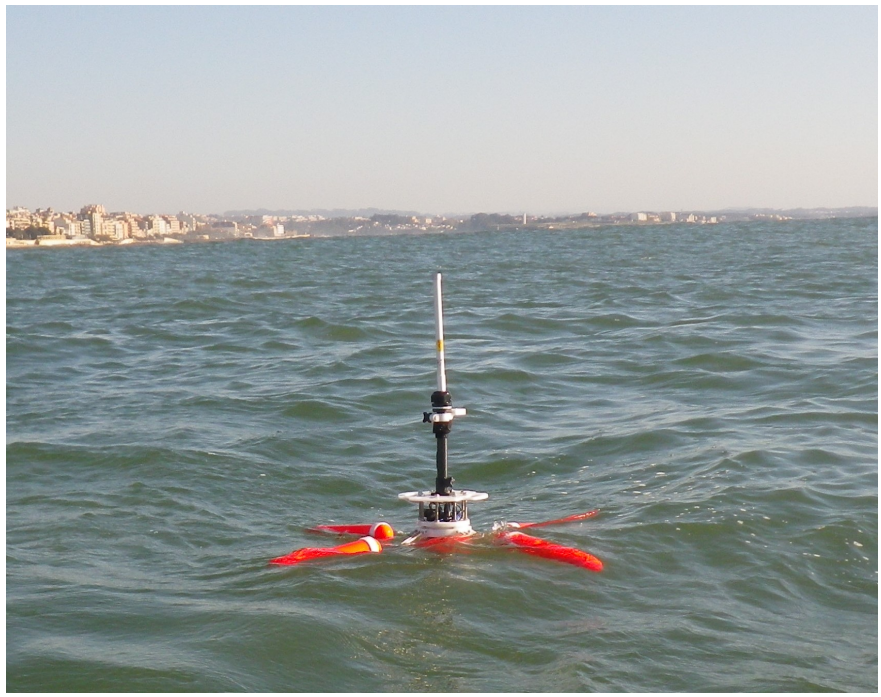

Fig. 1. Portable Acoustic Navigation Buoy deployed off Matosinhos, Porto, Portugal (February 2016).

and setup, the burden of transporting, deploying and setting up a network of moored beacons can easily become a significant portion of the whole mission time. Moreover, in the case of the new trend of man portable AUVs, the main requirement driving the size of the support vessel is no longer the size of the AUV, but, instead, the amount of people required for supervising the operation and the support devices that are needed. For many of these short term missions, some of the characteristics of the buoys may be sacrificed to gain on logistics, providing they can ensure their main job as positioning devices. Therefore, for a great number of missions, a small size fast deployment and recovery beacon is the ideal choice.

The remainder of this paper is organized as follows. Section II details the main requirements set for the development of this new generation of lightweight navigation beacons. Section III describes the main components of the buoy, namely in terms of hardware subsystems and general software architecture. Section IV explains the typical modes of operation for the navigation of AUVs, with some preliminary results of deployment in coastal waters in section V. Finally, section VI concludes the paper with a summary of the main conclusions and pointers for future work. 


\section{REQUIREMENTS}

Given our focus in the development of a new generation of small, man portable, navigation beacons, the main challenge was to ensure that the functional characteristics of the beacons were not compromised as we tried to fit them in a proper size and shape packaging. Following a systems engineering approach, the main requirements were identified for the buoy subsystems:

- Stability, size, and shape - The mechanical stability of a surface acoustic navigation beacon is important to ensure that any airborne antenna remains within the appropriate range of tilt angle. Similarly, the underwater transducer should maintain its orientation during a mission for a proper transmission of acoustic signals. Traditional buoys rely largely on size to ensure stability at sea. Naturally, larger buoys are easier to stabilize at sea, so one of the main challenges of this new design was to provide a similar stability as in a standard buoy, but with much less footprint for transportation.

- Energy management -As a side benefit of having a large structure, traditional buoys can house a significant amount of energy and they also have room to install energy harvesting devices. In our case, the sole requirement was for the beacon to last for at least one day of operations. To support multiple day operations the buoys should have rechargeable batteries and it should be possible to recharge them with minimum intervention.

- Computational System - In order to be able to support more advanced positioning or tracking approaches the buoy should house, at least, a small size computational system. This system should be able to time stamp and log all the data collected by the buoy, as well as to manage all internal devices.

- Communications - Communication with the computational system should be assured for long ranges, preferably above $1 \mathrm{~km}$. It was also desirable to have a high speed communication to allow the fast download of data, at least for short range. The real time link should allow to reconfigure the buoy during its deployment.

- Payload - The main subsystem of the new beacon is the set of underwater acoustic transmitters and receivers already implemented in traditional buoys. Many schemes of underwater positioning or tracking require or benefit from GPS georeferencing and synchronous clocks, therefore it should also be fitted with a GPS receiver with PPS. The GPS position can be used to update the buoys position, both in real time or in post processing, to improve the accuracy of vehicles navigation.

\section{System Description}

\section{A. Mechanical Hardware}

Our acoustic navigation buoys (fig.2) are composed of a poly-carbonate cylinder shaped water proof housing with an outside diameter of $120 \mathrm{~mm}$, containing all the electronic systems. All the electronics and trimming components were

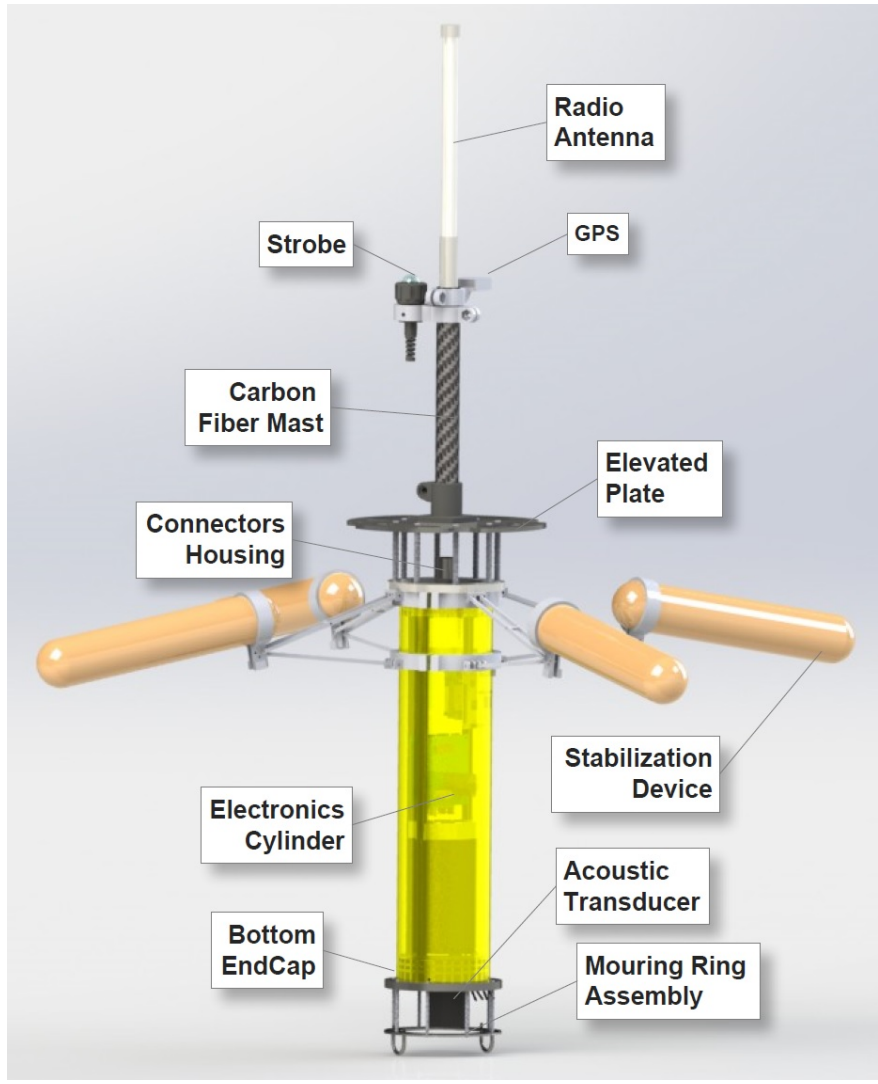

Fig. 2. Buoy mechanics description

placed in such a way that the cylinder is stable in the vertical position. Upon deployment this places one end-cap of the cylinder underwater and the other above the water line. The top end-cap features an elevated plate supporting a mast assembly with a radio and GPS antennas and a signaling light. The plate presents hand size grooves for facilitating the handling of the buoy. On the bottom end-cap a mooring ring assembly both forms a protective cage for an underwater transducer and holds two eye bolts for securing a mooring anchor.

For stability proposes, an umbrella like mechanical assembly was fitted into the buoy cylinder. Upon entering the water the system automatically extends four stabilization floats 30 centimeters from the cylinder geometric center. Along with the desired stability, this feature also adds some extra flotation to the overall system. The mechanism is composed of two discs connect to each other by an articulated system of stainless steel rods. The top ring is secured to the cylinder and the bottom one is left unrestrained. The stabilization floats are secured to the middle point of the articulated system. When the buoy enters the water, the flotation forces the bottom ring to travel upwards along the cylinder forcing the articulated system to fold like an elbow, extending floats away from the buoys geometric center. Figure 3 illustrates this mechanism by depicting the buoy in three different stages, from fully folded to fully extended. 


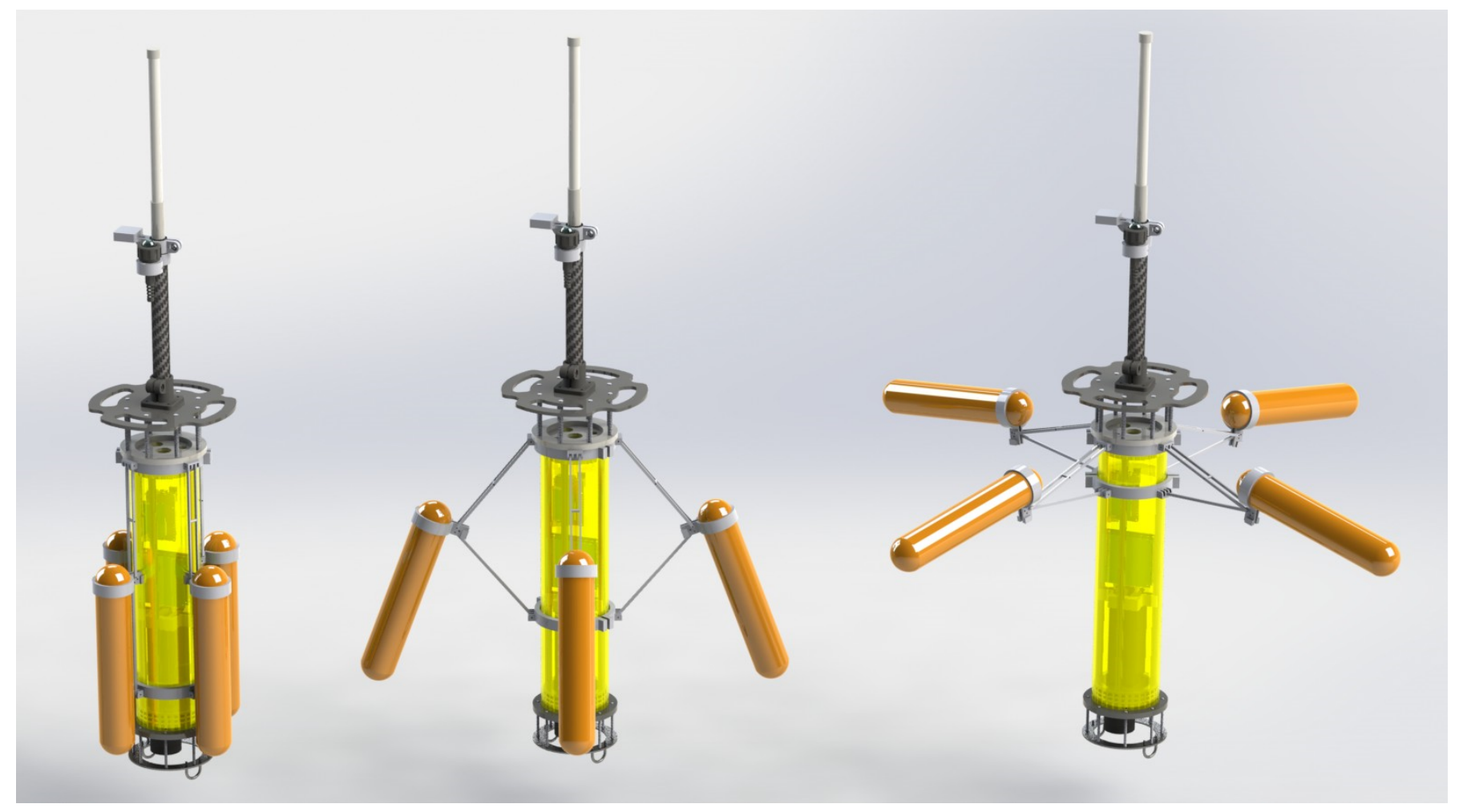

Fig. 3. Illustration of the automatic unfolding of the stabilization mechanism, as the buoy enters the water.

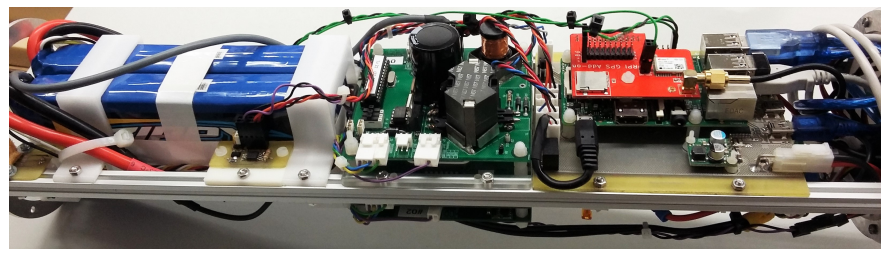

Fig. 4. Inside frame with electronics

\section{B. Electronics}

Inside the cylinder, it features an open frame (fig.4) securing a custom interface board with power level converters and electronic communications drivers for peripherals and sensors. Mechanically, the same board provides support to multiple subsystems like the main computational system, a GPS receiver and a serial radio modem. The space available allows to install any kind of small size embedded PC like an Odroid, BeagleBone or Raspberry Pi. In this work we used a Raspberry Pi V2 to demonstrated that even the simplest single board computer could fulfill all the requirements. As far as communications are concerned, there are two complementary mechanisms. A standard Wifi dongle connected directly to a USB port provides wide band connectivity up to $200 \mathrm{~m}$. For long range communications, a serial radio modem from Digi together with the external antenna, ensures connectivity for more than $1 \mathrm{Km}$, even though at only 9600 baud. To provide GPS data we used a simple GPS addon, fitted with a
Ublox Neo6 chip, that attaches directly onto the Raspberry's expansion header. This device provides a $5 \mathrm{~Hz}$ update rate and a PPS signal required for many navigation schemes. Our standard stack of proprietary acoustic boards was secured directly to the frame. This means that all acoustic features that we demonstrated in other buoys and vehicles remain available in this new generation of beacons. In particular, it supports various schemes of acoustic positioning such as long baseline using two way or one way travel time (TWTT/OWTT), like described in [2] and [4]. A digital compass was also included to measure the systems oscillation during a deployment. Finally to power the entire system a pack of Li-Po rechargeable batteries was installed in the frame. These are connected to a power management board that is responsible for switching the power to the system, monitoring the batteries status and protecting against any over-current. The board is also connected to an underwater connector on the top end cap, allowing to plug an external Li-Po charger to charge the batteries with minimum intervention. A diagram of the arrangement of the systems electronics can be seen in Figure 5.

\section{Software}

Our system features an embedded PC running a distribution of Linux and a stack of proprietary software running on it. This software stack is group of layered modules responsible for interfacing with all the peripheral, logging all the data and handling communications with other systems and control stations. Inter-module communications rely on a message passing mechanism implemented over UDP sockets, using 


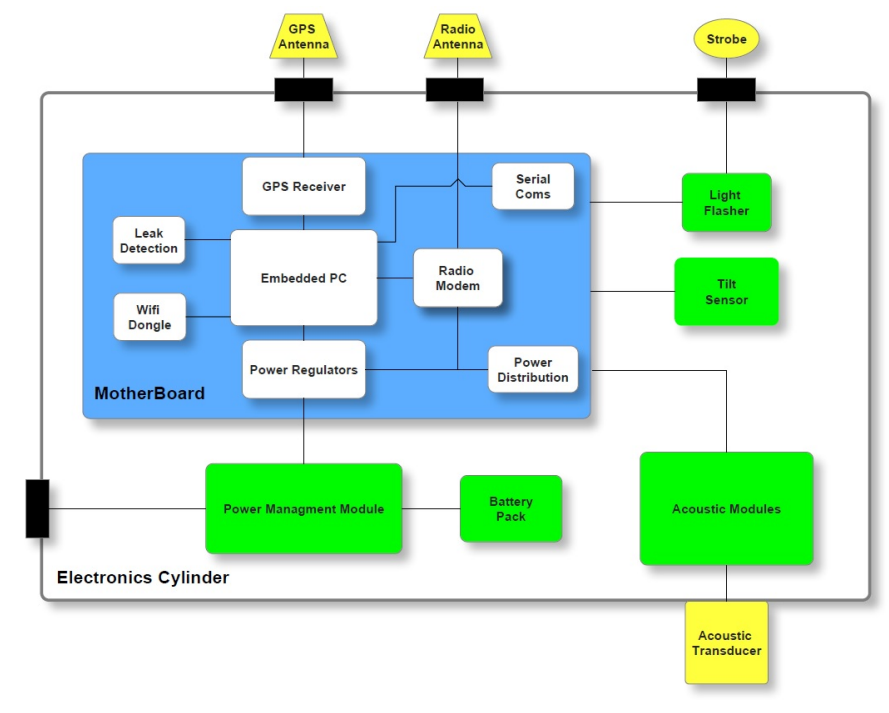

Fig. 5. Electronics cylinder diagram

TABLE I

BUOY SPECIFICATION

\section{General Specification}

Fast deploymen

Light weight

Total height

(30 $\mathrm{cm}$ mast and $7 \mathrm{~dB}$ antenna)

Diameter (Folded/Extended)

Rechargeable LiPo cells pack

Long range radio link

WiFi link

Acoustic link

GPS

\section{Energy Specifications}

$\begin{array}{lc}\text { Available energy } & 185 \mathrm{Whr} \\ \text { Average power consumption } & \simeq 6 \mathrm{~W} \\ \quad \text { Transponder or Transmitter mode @ } 1 \mathrm{~Hz} & +0,8 \mathrm{~W} \\ \text { Endurance } \quad \text { Tracking or OWTT Receiver } & \simeq 30 \mathrm{~h} \\ \text { Transponder or OWTT Transmitter @ } \frac{1}{2} \mathrm{~Hz} & \simeq 28 \mathrm{~h} \\ \text { Transponder or OWTT Transmitter @ } 1 \mathrm{~Hz} & \simeq 26 \mathrm{~h}\end{array}$

our proprietary protocol. The bottom layer of this stack is constituted by a series of processes, each one interfacing with a different sensor or peripheral, allowing for its configuration and creating an abstraction layer. Currently, the processes that compose this layer interface with the GPS receiver, with the acoustic system, and with a digital compass. Processes that implement modes of operation or internal monitoring activities constitute the high level layer. The selection of the processes that constitute this layer depends on the specific configuration of the buoy. Besides this layered organization, a dedicated module implements a data logging mechanism, receiving data from all interface and high level modules. This process also forwards data to external systems according to predefined rules, or based on a publisher-subscriber mechanism. Logged data can be easily exported to multiple formats, including MATLAB. Communication with external devices relies both
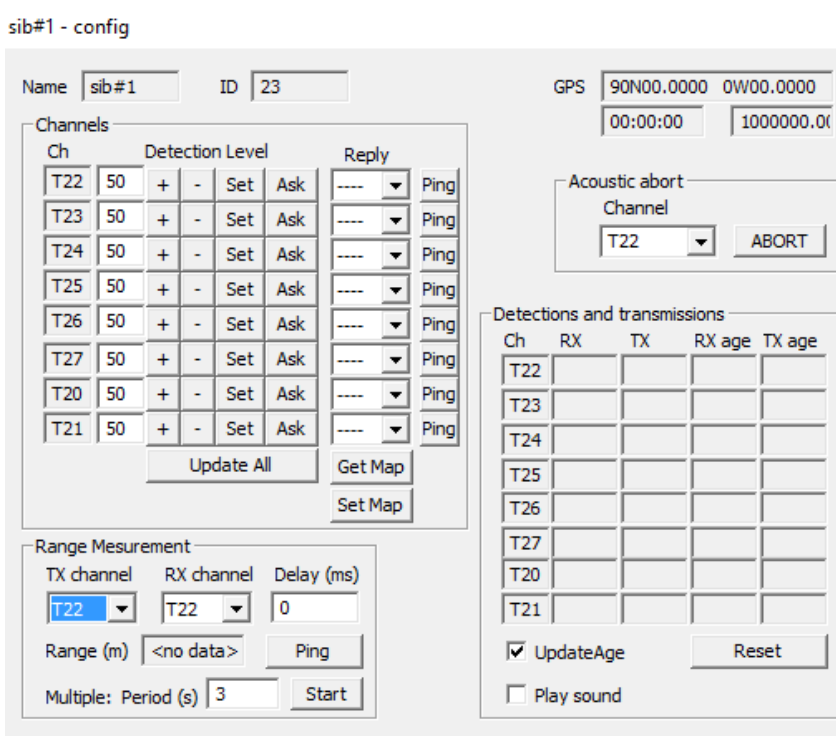

Detections and transmissions

\begin{tabular}{|c|c|c|c|c|}
\hline Ch & $\mathrm{RX}$ & $T X$ & RX age & TX age \\
\hline \multicolumn{5}{|l|}{$T 22$} \\
\hline \multicolumn{5}{|l|}{$\sqrt{T 23}$} \\
\hline \multicolumn{5}{|c|}{$\longdiv { \mathrm { T } 2 4 }$} \\
\hline \multicolumn{5}{|c|}{$\longdiv { T 2 5 }$} \\
\hline \multicolumn{5}{|l|}{$\overline{T 26}$} \\
\hline \multicolumn{5}{|c|}{ T27 } \\
\hline \multicolumn{5}{|l|}{$T 20$} \\
\hline \multicolumn{5}{|l|}{$\overline{T 21}$} \\
\hline \multicolumn{3}{|c|}{$\sqrt{\checkmark}$ UpdateAge } & \multicolumn{2}{|c|}{ Reset } \\
\hline$\Gamma \mathrm{p}$ & ay sound & & & \\
\hline
\end{tabular}

Fig. 6. Acoustic navigation buoy user interface

on a WIFI link (for short-range communications) and on serial radio link (for long-range communications). WIFI communication directly uses our proprietary protocol over UDP, while for serial communication, special interface processes run at each end-point in order to make such link transparent to the user.

\section{OPERATION}

Our beacons can be used in a wide variety of positioning and tracking schemes, ranging from traditional LBL to target tracking. The system uses GPS time and PPS for time base, allowing the synchronization among various units and targets. This allows for not only LBL schemes with two-way-traveltime range measurements but also the use of one-way-traveltime approaches, as described in [4].

The beacons are programmed through our proprietary user interface and communication protocol. The interface presents the basic information about the beacons, like the ID, its GPS position and the state of the transmission and receptions of the acoustic channels (fig.6).

The interface allows the user to check and set the detection levels for all acoustic channels, as well as to program a response map for two-way-travel-time acoustic range measurements. Also it's possible to take single or multiple range measurements to other units by defining a suitable transmission/reception map for two-way-travel-time or simply a transmission channel for one-way-travel-time. For multiple range measurements its also possible to define the periodicity of the acoustic pings.

\section{Field Performance}

\section{A. Deployment and Recovery}

The lightweight nature of the buoys allows them to be deployed by only one person. Also, a small number of them can easily be transported at once in a small support boat. 


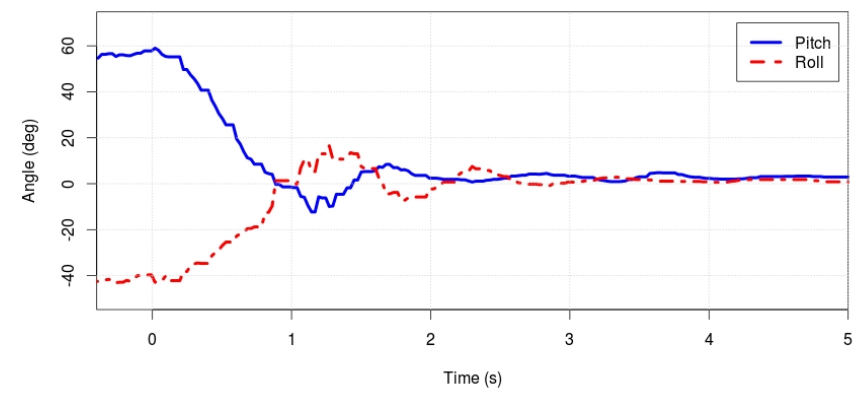

Fig. 7. Buoy stabilization when released with a pitch angle of 60 degrees and a roll angle of 40 degrees.

Depending on the scheme being used at the time, they can either be moored or left drifting.

When entering the water the flotation forces the stabilization system to open ensuring the upright position of the buoy, as illustrated in Figure 3. The system also dampens the tilting motion that follows the deployment. The beacon was intended to be deployed with sea state up to 2 . In rougher conditions, this system will soften the tilting motion caused by the waves, increasing the overall stability. Figure 7 shows the behavior of the buoy when released with a pitch angle of approximately 60 degrees and a roll angle of approximately 40 degrees. It is clear that the oscillation diminishes to negligible values in a time constant of 2 seconds

Recovery can also be performed by one person using a small boat. The hand size grooves in the elevated plate can be used to secure the buoy and pull it aboard. As the buoy exits the water the stabilization system will automatically close, decreasing its diameter, which will facilitate the recovery procedure.

\section{B. Endurance}

The power consumption during a deployment will vary depending on the type of application. This variation of the systems endurance is mostly related with the power consumption of the acoustic modules. Which depends whether it is being used for listening or transmitting acoustic signals. Naturally the duration of the acoustic signals and the transmission rate will also have an impact on the systems endurance, as seen in table I.

For the described scenarios the overall systems endurance is well inside the desired "day of operation" requirement. Even in the worst case scenario the batteries won't fully discharge during that time. Charging of the batteries is done with an external 50W LiPo Charger/Controller through the underwater connector on the top end cap. A full charge takes about 3,5 hours, and in 1,5 hours a 50\% charge is achieved.

\section{Data Collection}

All the data collected by the buoy is geotagged, time stamped and logged in a SD card. If desired, the buoy can be

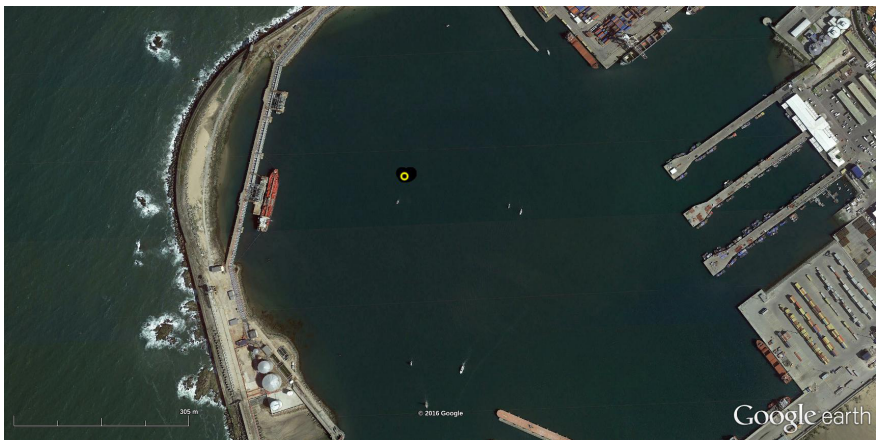

Fig. 8. GPS $\log$ example from a deployment at Leixões harbor, Porto, Portugal.

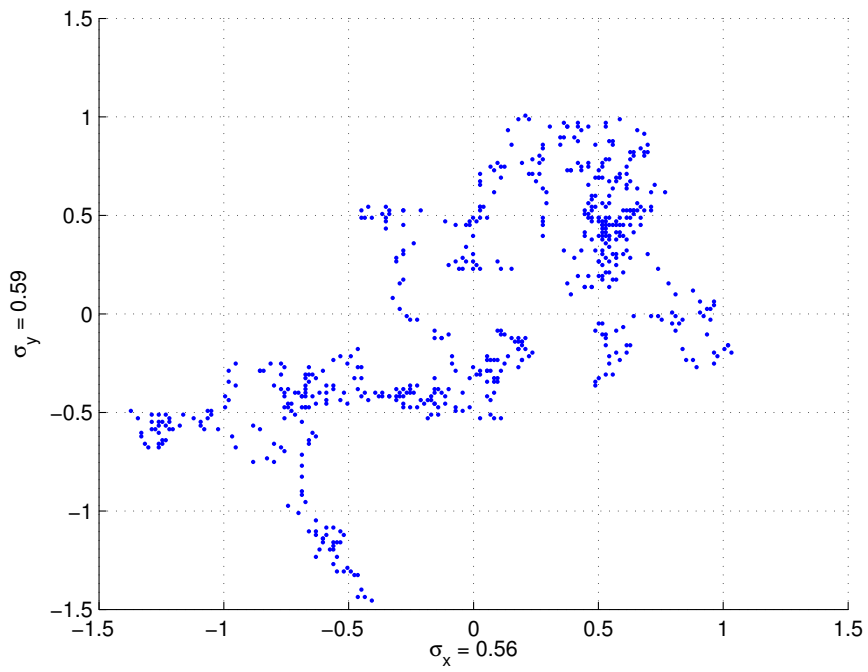

Fig. 9. Dispersion of the GPS measurements

configured to forward the data to a remote system where it can be stored or viewed in real time. This configuration, however can only be done through WiFi and should be done at close range. Figure 8 shows a KML plot generated from the GPS $\operatorname{logs}$ of a deployment carried out at Leixões harbor, Portugal. Figure 9 shows the dispersion of the GPS measurements, during approximately 30 minutes, in the deployment depicted in Figure 8. The deviation values are well inside the expected error for the GPS receiver.

\section{CONCLUSION}

This paper presented the development of a new generation of man portable buoys to support acoustic navigation of AUVs. The development strategy involved the identification of the vital requirements and then to pack the relevant modules into a very compact, yet dynamically stable, platform, allowing for one day long operations in coastal waters. All the requirements set in the beginning of the project were fully met, and we were able to integrate all the required system in a small size and lightweight buoy that can easily be deployed and recovery from sea by a single person. Moreover, given the reduced size of the mechanical assembly, with a mere $350 \mathrm{~mm}$ diameter 
footprint when folded, all operational logistics are simplified, allowing for a simple support boat to carry multiple beacons at once. Sea trials demonstrated that the buoy is mechanically robust and perfectly capable to perform in a typical operational scenario, including all features of previous implementations in much larger platforms, with the exception of autonomy. Throughout a mission, all payload data is stored on board, together with absolute time and GPS coordinates, and/or transmitted via radio. This is fundamental for AUV operations, since any position drift of the beacons can be transmitted to the vehicles in real time, or logged for post mission correction of measurements. In fact, this type of buoy was already used in a sea trial to collect data for the characterization of errors in acoustic positioning, as published in [4]. Apart from the main intention of acoustic beacons, there are other applications where these buoys can be used. For example, it is straightforward to deploy the buoys with no mooring and track their motion at the surface while receiving real time data from any on board sensor, either using the available communication devices or including other technologies, like Iridium. Although the size of the beacons is small, is is relatively simple to replace the sealing cylinder by a longer tube to accommodate additional payload and/or to provide extra stability.

\section{ACKNOWLEDGEMENTS}

This work is financed by the ERDF European Regional Development Fund through the Operational Programme for Competitiveness and Internationalisation - COMPETE 2020 Programme within project POCI-01-0145-FEDER-006961, and by National Funds through the FCT Fundao para a Ciłncia e a Tecnologia (Portuguese Foundation for Science and Technology) as part of project UID/EEA/50014/2013.

\section{REFERENCES}

[1] Cruz, N. and Madureira, L. and Matos, A. and Pereira, F.L., A versatile acoustic beacon for navigation and remote tracking of multiple underwater vehicles, OCEANS 2001 MTS/IEEE Conference and Exhibition, Honolulu, HI.

[2] Almeida, R. and Cruz, N. and Matos, A., Synchronized intelligent buoy network for underwater positioning,,OCEANS 2010 MTS/IEEE Conference and Exhibition, Seattle, WA.

[3] Thomas, H., GIB buoys: an interface between space and the depths of the oceans, in Proceedings of the 1998 IEEE Workshop in Autonomous Underwater Vehicles, Cambridge, MA.

[4] Almeida, R. and Melo, J. and Cruz, N., Characterization Of Measurement Errors In A LBL Positioning System, OCEANS 2016 MTS/IEEE Conference an Exhibittion, Shanghai, China. 